\title{
Microenvironment-related gene TNFSF13B predicts poor prognosis in kidney renal clear cell carcinoma
}

\author{
Mingzhe Jiang Equal first author, 1 , Jiaxing Lin ${ }^{\text {Equal first author, }{ }^{1} \text {, Haotian Xing }}{ }^{1}$, Jun An ${ }^{1}$, Jieping Yang ${ }^{1}$, Biao Wang ${ }^{2}$, Meng Yu $^{3}$, \\ Yuyan Zhu ${ }^{\text {Corresp. } 1}$ \\ ${ }^{1}$ Department of Urology, The First Hospital of China Medical University, Shenyang, Liaoning, China \\ 2 Department of Biochemistry and Molecular Biology, School of Life Sciences, China Medical University, Shenyang, Liaoning, China \\ 3 Key Laboratory of Transgenetic Animal Research, Liaoning Province, Department of Laboratory Animal Science, China Medical University, Shenyang, \\ Liaoning, China \\ Corresponding Author: Yuyan Zhu \\ Email address: yyzhu@cmu.edu.cn
}

Background Kidney renal clear cell carcinoma (KIRC) affects the genitourinary system. Although treatment of KIRC in early stages can be highly successful, this type of cancer is difficult to detect until later stages of disease that are less easily treatable. Previous studies have focused on tumor cells, but have ignored the contributions of the tumor microenvironment. Methods We analyzed KIRC gene expression data from The Cancer Genome Atlas with the ESTIMATE algorithm to identify differentially expressed genes. Through protein-protein interaction network analysis, we identified clusters and picked genes from the clusters for further analysis. Differential expression, Kaplan-Meier, and univariate Cox analyses were used to select prognostic biomarkers. Gene Set Enrichment Analysis (GSEA) and Tumor Immune Estimation Resource (TIMER) analysis were used to explore the immune characteristics of these genes as biomarkers. Results Through ESTIMATE algorithm and other system biology tools, TNFSF13B was identified as a prognostic biomarker. TNFSF13B is highly expressed in tumors, and high expression of TNFSF13B leads to poor prognosis. Further GSEA and TIMER analysis revealed that the expression of TNFSF13B was related to immune signaling pathway and lymphocyte infiltration. Our findings strongly suggest that TNFSF13B may be a potential biomarker or target related to the tumor microenvironment for KIRC. 
1 Microenvironment-related gene TNFSF13B predicts poor prognosis in kidney renal clear 2 cell carcinoma

3 Mingzhe Jiang ${ }^{1 *}$, Jiaxing Lin ${ }^{1 *}$, Haotian Xing ${ }^{1}$, Jun An ${ }^{1}$, Jieping Yang ${ }^{1}$, Biao Wang ${ }^{2}$, Meng $4 \mathrm{Yu}^{3}$, Yuyan Zhu ${ }^{1 \#}$

51 Department of Urology, The First Hospital of China Medical University, Shenyang, Liaoning, 6 China

72 Department of Biochemistry and Molecular Biology, School of Life Sciences, China Medical 8 University, Shenyang, Liaoning, China

93 Key Laboratory of Transgenetic Animal Research, Liaoning Province, Department of

10 Laboratory Animal Science, China Medical University, Liaoning, Shenyang, Liaoning, China

11 *Mingzhe Jiang and Jiaxing Lin contributed equally to this work

12 \# Corresponding Author: Yuyan Zhu, No. 155 Nanjing North Street, Heping District, Shenyang,

13 Liaoning, 110001, China.

14 Email address: yyzhu@cmu.edu.cn

\section{Abstract}

\section{Background}

17 Kidney renal clear cell carcinoma (KIRC) affects the genitourinary system. Although treatment 
18 of KIRC in early stages can be highly successful, this type of cancer is difficult to detect until

19 later stages of disease that are less easily treatable. Previous studies have focused on tumor cells,

20 but have ignored the contributions of the tumor microenvironment.

\section{Methods}

22 We analyzed KIRC gene expression data from The Cancer Genome Atlas with the ESTIMATE 23 algorithm to identify differentially expressed genes. Through protein-protein interaction network 24 analysis, we identified clusters and picked genes from the clusters for further analysis. 25 Differential expression, Kaplan-Meier, and univariate Cox analyses were used to select 26 prognostic biomarkers. Gene Set Enrichment Analysis (GSEA) and Tumor Immune Estimation 27 Resource (TIMER) analysis were used to explore the immune characteristics of these genes as 28 biomarkers.

\section{Results}

Through ESTIMATE algorithm and other system biology tools, TNFSF13B was identified as a

31 prognostic biomarker. TNFSF13B is highly expressed in tumors, and high expression of

32 TNFSF13B leads to poor prognosis. Further GSEA and TIMER analysis revealed that the expression of TNFSF13B was related to immune signaling pathway and lymphocyte infiltration.

Our findings strongly suggest that TNFSF13B may be a potential biomarker or target related to the tumor microenvironment for KIRC.

\section{Introduction}


37 Kidney renal clear cell carcinoma (KIRC) is a highly prevalent urinary system cancer,

38 accounting for $80 \%$ to $90 \%$ of lesions in the renal space and about $3 \%$ of all new cases of tumors

39 worldwide. About 100,000 patients die of renal cell carcinoma every year (Siegel et al., 2017),

40 and KIRC is the main pathological type of this carcinoma. Although biotherapy and surgical

41 treatment can be effective in early stages, renal cell carcinoma typically is not detected until later

42 stages. Additionally, KIRC typically progresses rapidly and exhibits early metastasis, with

43 overall poor patient prognosis. Therefore, identification of KIRC-related genes can provide

44 insight into the mechanism of renal cell carcinoma and facilitate early diagnosis and treatment of

45 renal cell carcinoma (Cairns, 2010).

46 To correlate tumor genetic composition with clinical prognosis, extensive databases of genome-

47 scale gene expression data have been established. The Cancer Genome Atlas (TCGA, 48 https://portal.gdc.cancer.gov/) is a public available repository of cancer genomic data, with data

49 relevant to several important cancers. These data can be analyzed to detect genomic changes that

50 can provide insight into cancer development. The expression patterns of different genes can be

51 compared to clinical diagnostic criteria and may help to identify markers that are relevant to

52 cancer diagnosis, may facilitate selection of effective treatment options, and can improve

53 prognosis (Krishnan et al., 2016).

54 Previous studies have shown that tumor cell intrinsic genes determine the occurrence, 55 development, and evolution of KIRC (Hsieh et al., 2017). However, the tumor microenvironment 56 generally has a serious impact on gene expression in cancer, as well as in KIRC and other solid 
57 tumor tissues, which can affect clinical consequences (Chevrier et al., 2017). The tumor 58 microenvironment is complex, and includes inflammatory cytokines and mediators, extracellular 59 matrix (ECM) molecules, and immune, mesenchymal, and endothelial cells (Hanahan \& 60 Coussens, 2012).

61 Of these components, immune and stromal cells are likely the key contributors to the tumor 62 microenvironment, and understanding how these cells affect tumor development is essential for 63 tumor diagnosis and assessment of prognosis. "Estimation of STromal and Immune cells in 64 MAlignant Tumours using Expression data" (ESTIMATE) is a method that uses gene expression 65 signatures to infer the level of non-tumor cells in tumor samples (Yoshihara et al., 2013). The 66 algorithm is based on single sample gene set enrichment analysis, and the levels of immune cells 67 and stromal cells are inferred by calculating immune score and matrix score. The ESTIMATE 68 scores reflect the purity of tumor cells, where the higher the ESTIMATE scores, the lower the 69 tumor purity. Previous studies have effectively used the ESTIMATE algorithm to analyze large 70 datasets to provide insight into melanoma (Yang et al., 2019), breast cancer (Chen et al., 2019), 71 and bladder cancer (Wang et al., 2019). However, despite numerous studies showing the efficacy 72 of this data-based algorithm and the ability to calculate immune and stromal scores for KIRC, no 73 detailed investigation has been conducted.

74 In this first report using the TCGA database to assess the KIRC cohort using the ESTIMATE 75 algorithm, we identified the gene related to the microenvironment that should be further 76 characterized for its ability to predict the prognosis of patients with KIRC. 
Materials and methods

\section{Database}

79 The work flow of this study is shown in Fig. 1. The expression profiles FPKM (level 3) and

80 clinical data were obtained for KIRC patients using the TCGA website in March 1, 2019

81 (https://portal.gdc.cancer.gov/). The immune, stromal, and ESTIMATE scores of each sample

82 were determined according to the ESTIMATE algorithm. This tool uses expression data to

83 estimate the contributions of stromal and immune cells in malignant tumor tissue.

84 Identification of differentially expressed gene (DEGs).

85 The package "limma" (https://bioconductor.org/packages/limma/, version=3.42.2) was used for

86 expression data analysis (Ritchie et al., 2015). The input data are the expression data of KIRC.

87 According to the median value of the scores, the patients were divided into two groups for

88 difference analysis. We then used Wilcoxon test to test the difference in the expression of each

89 gene between the two groups. $|\log \mathrm{FC}|>1$ (FC: fold change) and false discovery rate (FDR) $\mathrm{p}<$

$90 \quad 0.05$ were set as the cutoff values to screen DEG.

$91 \quad$ Heatmap and cluster analysis

92 The R package "pheatmap" (https://CRAN.R-project.org/package=pheatmap, version=1.0.12)

93 was used to generate a heatmap and perform cluster analysis. The gene expression profile data

94 were input, the gene expression heat map was drawn, and the genes were clustered according to 95 the sample groups. 
96 Gene ontology (GO) and Kyoto Encyclopedia of Genes and Genomes (KEGG) pathway

97 analysis

98 The GO and KEGG analyses were performed using the R package "clusterProfiler"

99 (https://bioconductor.org/packages/clusterProfiler/, version=3.14.3) (Yu et al., 2012). FDR p

100 value $<0.05$ was used to distinguish significantly enriched terms. Enter the genes, and used the

101 functions "enrichGO" and "enrichKEGG" for enrichment analysis. The results of enrichment

102 analysis are shown by bubble diagram.

Construction of protein-protein interaction (PPI) network and identification of functional

clusters

The PPI network was constructed using the Search Tool for the Retrieval of Interacting Genes

(STRING; https://string-db.org/) (Szklarczyk et al., 2019). Unconnected genes were removed and the confidence was set to 0.4 to obtain the PPI network. We further analyzed the PPI network with Cytoscape (Cytoscape_v3.6.1) (Shannon et al., 2003) and then Molecular Complex Detection (MCODE) was used to identify functional clusters (Bader \& Hogue, 2003). The

110 default parameters were set as follows: the degree cutoff value was 2 , the node score was 0.2 , the

$111 \mathrm{k}$-score was 2, and the maximum depth was 100.

\section{Kaplan-Meier survival analysis}

113 Kaplan-Meier curve has time as the horizontal axis and survival rate as the vertical axis. The $\mathrm{R}$

114 packages "survival" (https://CRAN.R-project.org/package=survival, version=3.1-8) and 
115 "survminer" (https://CRAN.R-project.org/package=survminer, version=0.4.6) were applied for

116 survival analysis. A value of $p<0.05$ means it is statistically significant. The input matrix

117 contained the sample's survival time, survival status, and gene expression data. Samples were

118 grouped using a median or optimal threshold for Kaplan-Meier regression analysis.

\section{Univariate and multivariate Cox regression analysis}

120 Cox analysis is a semi-parametric model of survival analysis, which is used to identify the risk

121 factors of disease and to analyze prognosis. Univariate and multivariate Cox analysis were 122 performed with the R package "survival". The input data was the same as the Kaplan-Meier

123

124

125

126 analysis, which was calculated using the function "cox" in package. The results of the analysis can be displayed in a forest map.

\section{Identification of practical prognostic biomarker}

Three methods were used to select practical prognostic biomarker. The first was to find out that the DEGs between tumor and normal tissue, the threshold was set to $\mid \log$ FC $\mid>1$, FDR-p $<$ 0.05; the second was to use univariate Cox analysis to get the prognostic genes $(\mathrm{p}<0.05)$; the third was to use Kaplan-Meier analysis to find out the prognostic genes $(p<0.05)$. Finally, the intersection of the three methods results was taken, and the Venn diagram is drawn with a Web tool to show (http://bioinformatics.psb.ugent.be/webtools/Venn/). We used DEG, and univariate COX here to screen prognostic markers for KIRC, and we did so because this is the most common way to obtain prognostic markers. We added Kaplan-Meier analysis because it can 
134 intuitively evaluate the prognosis of patients and has practical significance for clinical use. With

135 the intersection of the three meaningful results, practical prognostic markers can be obtained.

136 Gene set enrichment analysis

137 Gene Set Enrichment Analysis (GSEA) is a computational method that determines whether a 138 group of basically defined genes exhibit statistically significant differences between two 139 biological states (Aravind et al., 2005). In this study, according to the gene expression data, the 140 samples were divided into two groups for GSEA. One thousand genome replacement tests were 141 performed per analysis in GSEA_4.0.1 and the expression level of each individual gene was used 142 as a phenotypic tag. Values of $p$-value $<0.05$ and FDR p-value $<0.05$ were applied to define the 143 pathways with statistical significance. Finally, we used the R package "ggplot2" 144 (https://CRAN.R-project.org/package=ggplot2, version=3.2.1) to draw multiple pathways for 145 display, the input file is the result of GSEA's analysis..

146 Exploration of immune characteristics and drug targets

147 The R package "ggstatsplot" (https:/CRAN.R-project.org/package=ggstatsplot, version=0.4.0) was used to analyze the correlation between genes expression and the scores. We used the Tumor Immune Estimation Resource (TIMER; https://cistrome.shinyapps.io/timer/) (Li et al., 2017) to explore the relationship between genes and immune cells infiltration. Finally, we queried the Tumor Immune System Interactions Database (TISIDB; http://cis.hku.hk/TISIDB) (Ru et al., 2019) for drugs that can act on the genes, using the drug information in the database from 


\section{Statistical Analysis}

155 All statistical analyses, including the operation of the ESTIMATE algorithm and gene

156 identification, were run using $\mathrm{R}$ software ( $\mathrm{R} \times 64$ 3.5.1). All $\mathrm{R}$ packages were obtained from

157 CRAN (https://cran.r-project.org) and Bioconductor (http://www.bioconductor.org). The two

158 groups were compared by the Wilcoxon test, and comparison between multiple groups was performed by Kruskal-Wallis test. Statistical significance was defined as $\mathrm{p}<0.05$.

160

161

162

\section{Results}

\section{Scores of the ESTIMATE algorithm are related to the survival and prognosis of kidney renal clear cell carcinoma}

The gene expression profiles and clinical data were obtained for all KIRC patients available in the TCGA database. Using the ESTIMATE algorithm, the stromal score range was -1433.77 to 1967.19, the immune score range was -693.96 to 3328.21, and the ESTIMATE score range was 2127.72 to 5091.59. To make the Kaplan-Meier analysis more statistically significant, the function "res.cut" in $\mathrm{R}$ package "survminer" was used to find the best segmentation point to group the samples. The scores of each group were grouped according to the optimal segmentation point calculated by the function. The best segmentation point based on immune score was 1778.92 . Relative to this point, the samples were divided into high and low immune score groups for Kaplan-Meier overall survival analysis. The results showed that the prognosis of 
172 the high immune score group was worse than the overall group $(p=0.003$, Fig $2 A)$. In a similar

173 analysis, the best segmentation points for stromal and ESTIMATE scores were 830.37 and

174 2785.69, respectively. Kaplan-Meier analysis showed worse prognosis of the high score group

175 for both stromal and ESTIMATE score-based analysis ( $<<0.05$, Fig $2 \mathrm{~B}-3 \mathrm{C})$. This shows that all

176 three scores predict the overall survival rate of patients. We generated a boxplot to show the

177 relationships between the three scores and pathological stages of KIRC, and then analyzed the

178 statistical significance by Kruskal-Wallis test. The results showed that the immune scores (Fig.

179 2D, $p<0.001$ ) and ESTIMATE scores (Fig. 2F, p =0.006) were both significant in different

180 pathological stages, and increased with the increase of pathological stages. However, there were

181 no differences in the stromal scores among different stages (Fig. 2E, $p=0.140$ ).

182

183

184

185

186

187

188

189

190

191

Mining differential genes in gene expression profiles according to immune score and stromal score

539 KIRC samples were first grouped according to the median of immune score and then a heatmap of gene expression was constructed (Fig. 3A). In the differential gene analysis of immune score, 512 genes were up-regulated and 147 genes were down-regulated $(|\log \mathrm{FC}|>1$, p

$<0.05)$. Similarly, the samples were grouped according to the median of stromal score and constructing heat maps of gene expression (Fig. 3B). 259 genes were up-regulated and 152 genes were down-regulated in the groups with high and low stromal scores $(|\log \mathrm{FC}|>1, \mathrm{p}<0.05)$. Since both immune and stromal factors are part of the immune microenvironment, we took the intersection between immune up-regulated genes and stromal up-regulated genes. Then we also 
192

193

194

195

196

197

198

199

200

201

202

203

204

205

206

207

208

209

210

took the intersection between immune down-regulated genes and stromal down-regulated genes.

The Venn map shows that 48 genes are generally up-regulated (Fig. 3C) and 47 genes generally are down-regulated (Fig. 3D). We decided to focus on these 95 DEGs, which we propose are genes that are important to the microenvironment. We investigated the function of these genes by GO and KEGG enrichment analysis. The most significant term of GO enrichment analysis is Humoral immune response, while that of KEGG enrichment analysis is Cytokine-cytokine receptor interaction. These results suggest that these genes are related to immunity (Fig. 3E-3F).

\section{Construction of PPI network and identification of functional clusters}

To better understand the interaction of DEGs in the microenvironment, we used the STRING tool to construct a PPI network for the 95 DEGs and visualize the network with Cytoscape (Fig. 4A). The network consists of 53 nodes and 110 edges, and 42 genes are not displayed in the network because they are not linked to the other genes. We then used the tool MCODE of

Cytoscape to identify the functional clusters; this tool aggregates highly related genes into a cluster. A total of four clusters which contain 24 genes were identified (Fig. 4B-4F). We took the 24 genes for the next step of analysis.

\section{Identification of KIRC practical prognostic biomarker}

We used three methods to screen practical prognostic biomarkers from the 24 genes. The expression profiles of 24 genes were analyzed to find the DEGs of tumor and normal tissues. A total of seven DEGs (CD163, CCL18, TNFSF13B, IL7R, IL2RA, PROZ, and PAH) were found 
$211(|\log \mathrm{FC}|>1, \mathrm{p}<0.05)$. According to the median expression of each gene, the genes were divided

212 into high expression group and low expression group. Kaplan-Meier analysis of the 24 genes

213 showed that seven genes (TNFSF13B, XCR1, IGLL5, PAH, IL10, F7, and AMBP) showed

214 significant differences in expression $(\mathrm{p}<0.05)$. Univariate Cox analysis of the 24 genes showed

215 that expression levels of 11 genes (CCL13, IL2RA, TNFSF13B, IGLL5, CCL19, SPIB, CCL21,

216 CR1, TNFRSF17, CD19, POU2AF1) were significantly different $(\mathrm{p}<0.05)$. The results of the

217 three analyses were combined in a Venn diagram (Fig. 5). TNFSF13B is the only gene found in 218 all three analysis.

\section{High expression of TNFSF13B leads to poor prognosis of KIRC}

220 The expression of TNFSF13B in tumor tissue is higher than that in normal tissue $(\mathrm{p}<0.001$, Fig.

221 6A). High expression of TNFSF13B leads to poor prognosis of KIRC ( $p=00019$, Fig. 6B). The

222 clinical factors and TNFSF13B were analyzed by univariate and multivariate regression analysis.

223 Univariate Cox analysis showed that Age, Grade, Stage, T (T stage), N (Lymph node), M

224 (Tumor metastasis), and TNFSF13B $(\mathrm{p}<0.001)$ were prognostic factors (Fig. 6C), it has very

225 significant statistical significance. Multivariate Cox analysis showed that Grade and Age were

226 independent prognostic factors (Fig. 6D). The result indicated that TNFSF13B was a prognostic

227 factor, but it could not analyze the prognosis independently of other clinical factors. The

228 relationship between gene expression and stage/grade was analyzed by box diagram, and

229 Kruskal-Wallis test was used for comparison among groups. The results showed that the

230 TNFSF13B expression was different in different stages and grades $(\mathrm{p}<0.001$, Fig. 6E-6F), and 
231 the TNFSF13B expression increased with the increase of stage and grade.

232 There is a positive correlation between TNFSF13B and tumor microenvironment.

233 By analyzing the correlation between immune/stromal/ESTIMATE score and TNFSF13B 234 expression, it was found that TNFSF13B was positively correlated with immune $(\mathrm{R}=0.67, \mathrm{p}<$ 235 0.001, Fig 7A), stromal $(\mathrm{R}=0.43, \mathrm{p}<0.001$, Fig 7B), and ESTIMATE $(\mathrm{R}=0.64, \mathrm{p}<0.001$, Fig

236 7C). We next queried the relationship between TNFSF13B and immune cell infiltration in the 237 TIMER database (Fig. 7D-7J), and found that its expression was positively correlated with tumor 238 purity and positively correlated with immune cells. TNFSF13B expression value showed 239 maximum partial spearmanm's correlation with dendritic cells ( partial cor $=0.634, \mathrm{p}=1.35 \mathrm{E}-52$ ).

\section{Gene set enrichment analysis of TNFSF13B}

241 GSEA is a powerful analytical method used to interpret gene expression data. We sequentially 242 analyzed gene enrichment and performed GSEA for TNFSF13B. We used the Molecular 243 Signatures Database (MSigDB, http://www.broadinstitute.org/msigdb) of Collection 244 (c2.cp.kegg.v7.0.symbols.gmt) (A Liberzon et al. 2011; A Liberzon et al. 2015). We set the 245 threshold for FDR p-value $<0.05$ and p-value $<0.05$. Significantly enriched signal transduction 246 pathways were identified based on normalized enrichment score (NES). We identified pathways with high NES that are related to immune function. Because few meaningful signal pathways were enriched by low gene expression, we only present the signal pathways enriched for high gene expression (Fig. 8A). There was clear enrichment in immune-related signaling pathways. 
250 Finally, we found three drugs that have effects on TNFSF13B in the TISIDB database (Fig. 8B),

1, 4-dimethyl Dioxide (DB03316), Citric Acid (DB04272), and Belimumab (DB08879). Among

them, Belimumab is an intravenous immunosuppressant and the only target for the adjuvant

treatment of systemic lupus erythematosus (SLE) (LJ Scott et al. 2012).

\section{Discussion}

255

256

257

Previous experiments of KIRC focused on animal tumor models, in vitro tumor cell lines, or patient tumor samples (Capitanio \& Montorsi, 2016). However, the KIRC microenvironment must also be considered. With increased exploitation of whole genome sequencing, TCGA has been established as an extensive tumor database for free use by the research community. These resources facilitate data analysis of many cancers, including KIRC (Krishnan et al., 2016).

The goal of this study was to identify genes that are related to the microenvironment and overall survival of KIRC. Gene expression patterns were compared for cases with high and low immune scores, and 95 genes were identified as potentially involved in functions related to the extracellular matrix or to immune response. To assess prognostic ability, we analyzed 95 differentially expressed genes from groups with high immune score (or high stromal score), and used GO and KEGG enrichment analysis to determine that many of these genes are components of the tumor microenvironment. Next, we constructed a protein-protein interaction network of these 95 genes, and identified four clusters which contained 24 genes. We performed differential expression, Kaplan-Meier, and univariate Cox analyses for the 24 genes. The TNFRSF13B gene was identified in all three analyses. TNFRSF13B was overexpressed in tumors, Kaplan-Meier 
270

271

272

273

274

275

276

277

278

279

280

281

282

283

284

285

286

287

288

289

analysis showed that high expression of TNFRSF13B led to poor prognosis, and univariate Cox showed that TNFRSF13B was a risk factor. The expression of TNFRSF13B is positively correlated with tumor stage and grade. These results suggest that TNFRSF13B is a potential prognostic marker.

TNFRSF13B is an essential factor in the tumor microenvironment of KIRC. There was a high positive correlation between TNFRSF13B and ESTIMATE score, indicating that the more TNFRSF13B expression, the less the content of tumor cells, the more the content of immune cells and stromal cells. Renal cell carcinoma has a good effect on immunotherapy (29120911), so we inquired about the relationship between TNFRSF13B and six kinds of immune cells. TNFRSF13B has a high correlation with immune cells. GSEA results showed that TNFRSF13B was involved in many immune-related pathways, including the $\mathrm{T}$ cell receptor signaling pathway and so on. TNFSF13B belongs to the tumor necrosis factor (TNF) ligand family, and is also named BAFF. TNFSF13B variants are associated with multiple sclerosis and SLE (Steri et al., 2017), and TNFSF13B is a key factor in the survival of B lymphocytes (Cremasco et al., 2014). Previous study suggested a potential role of these TNFSF members including TNFRSF13B in renal tumor biology (Pelekanou et al., 2011). Recently, it has been reported that TNFSF13B is related to the prognosis and immune infiltration of KIRC (Li et al., 2019). This article starts directly from TNFSF13B to explore its prognosis and immune infiltration. However, we started from the tumor microenvironment, went through many aspects of screening, and finally selected the representative gene TNFSF13B. Their research and our exploration have jointly confirmed 
290 the important role of TNFSF13B in KIRC. Through our research, TNFSF13B can not only

291 predict the prognosis of KIRC, but also be an important medium for KIRC to transmit

292 information between the tumor cell and tumor microenvironment, and is a potential therapeutic

293 target.

294 The interaction between KIRC and the tumor microenvironment seriously affects the progress

295 and evolution of this carcinoma, and subsequently affects the recurrence and overall prognosis of

296 patients. Previous reports have provided a detailed analysis of how the activation of tumor

297 intrinsic genes shapes the tumor microenvironment. In our current work, we analyzed the genetic

298 characteristics of the microenvironment that affect the development of KIRC. Our results may

299 help explain the complex interactions between the tumor and the tumor environment in kidney

300 renal clear cell carcinoma. Of course, our research also has some shortcomings. In the future, we

301 need more data to prove the prognostic ability of TNFSF13B and also need experiments to prove

302 the relationship between TNFSF13B and microenvironment.

303 Conclusions

304 We analyzed KIRC gene expression data from The Cancer Genome Atlas with the ESTIMATE

305 algorithm to identify the differentially expressed genes. Through multiple analyses, we identified

306 TNFSF13B as a potential biomarker and target for kidney renal clear cell carcinoma. Further

307 study of TNFSF13B may improve understanding of the potential relationships between the tumor

308 microenvironment and the prognosis of kidney renal clear cell carcinoma. 
310 We appreciate the data obtained from the TCGA database in this study.

311 Abbreviations: KIRC - Kidney renal clear cell carcinoma, TCGA - The Cancer Genome Atlas,

312 ESTIMATE -using expression data to estimate stromal and immune cells in malignant tumor

313 tissue, DEGs - differentially expressed genes, FC - fold change, GSEA - Gene Set Enrichment

314 Analysis. FDR - false discovery rate, PPI - protein-protein interaction, TIMER - Tumor Immune

315 Estimation Resource.

316

317

318

319

320

321

322

323

324

325

326

327

328

329

330

331

332

333

\section{References}

Liberzon A, Subramanian A, Pinchback R, Thorvaldsdóttir H, Tamayo P, and Mesirov Jp. 2011. Molecular signatures database (MSigDB) 3.0. Bioinformatics (Oxford, England) 27:1739-1740. DOI 10.1093/bioinformatics/btr260

Liberzon A, Birger C, Thorvaldsdóttir H, Ghandi M, Mesirov Jp, and Tamayo P. 2015. The Molecular Signatures Database (MSigDB) hallmark gene set collection. Cell systems 1:417-425. DOI 10.1016/j.cels.2015.12.004

Krishnan B, Rose Tl, Kardos J, Milowsky Mi, and Kim Wy. 2016. Intrinsic Genomic Differences Between African American and White Patients With Clear Cell Renal Cell Carcinoma. JAMA oncology 2:664-667. DOI 10.1001/jamaoncol.2016.0005

Ru B, Wong Cn, Tong Y, Zhong Jy, Zhong Ssw, Wu Wc, Chu Kc, Wong Cy, Lau Cy, Chen I, Chan Nw, and Zhang J. 2019. TISIDB: an integrated repository portal for tumor-immune system interactions. Bioinformatics (Oxford, England) 35:4200-4202. DOI 10.1093/bioinformatics/btz210

Wang B, Pan W, Yang M, Yang W, He W, Chen X, Bi J, Jiang N, Huang J, and Lin T. 2019. Programmed death ligand-1 is associated with tumor infiltrating lymphocytes and poorer survival in urothelial cell carcinoma of the bladder. Cancer Science 110:489-498. DOI 10.1111/cas.13887

Chen Ch, Lu Ys, Cheng Al, Huang Cs, Kuo Wh, Wang My, Chao M, Chen Ic, Kuo Cw, Lu Tp, and Lin Ch. 2019. Disparity in Tumor Immune Microenvironment of Breast Cancer and Prognostic Impact: Asian versus Western Populations. The oncologist undefined:undefined. DOI 10.1634/theoncologist.2019-0123 
334

335

336

337

338

339

340

Hanahan D, and Coussens Lm. 2012. Accessories to the crime: functions of cells recruited to the tumor microenvironment. Cancer Cell 21:309-322. DOI 10.1016/j.ccr.2012.02.022

Szklarczyk D, Gable Al, Lyon D, Junge A, Wyder S, Huerta-Cepas J, Simonovic M, Doncheva Nt, Morris Jh, Bork $\mathrm{P}$, Jensen $\mathrm{Lj}$, and Mering Cv. 2019. STRING v11: protein-protein association networks with increased coverage, supporting functional discovery in genome-wide experimental datasets. Nucleic Acids Research 47:D607-D613. DOI 10.1093/nar/gky1131

Yu G, Wang Lg, Han Y, and He Qy. 2012. clusterProfiler: an R package for comparing biological themes among gene clusters. Omics : a journal of integrative biology 16:284-287. DOI 10.1089/omi.2011.0118

Bader Gd, and Hogue Cw. 2003. An automated method for finding molecular complexes in large protein interaction networks. BMC Bioinformatics 4:2. DOI 10.1186/1471-2105-4-2

Hsieh Jj, Purdue Mp, Signoretti S, Swanton C, Albiges L, Schmidinger M, Heng Dy, Larkin J, and Ficarra V. 2017. Renal cell carcinoma. Nature reviews Disease primers 3:17009. DOI 10.1038/nrdp.2017.9

Yoshihara K, Shahmoradgoli M, Martínez E, Vegesna R, Kim H, Torres-Garcia W, Treviño V, Shen H, Laird Pw, Levine Da, Carter Sl, Getz G, Stemke-Hale K, Mills Gb, and Verhaak Rg. 2013. Inferring tumour purity and stromal and immune cell admixture from expression data. Nature Communications 4:2612. DOI $10.1038 /$ ncomms 3612

Scott Lj, Burness Cb, and McCormack Pl. 2012. Belimumab: a guide to its use in systemic lupus erythematosus. BioDrugs : clinical immunotherapeutics, biopharmaceuticals and gene therapy 26:195-199. DOI 10.2165/11209060-000000000-00000

Steri M, Orrù V, Idda Ml, Pitzalis M, Pala M, Zara I, Sidore C, Faà V, Floris M, Deiana M, Asunis I, Porcu E, Mulas A, Piras Mg, Lobina M, Lai S, Marongiu M, Serra V, Marongiu M, Sole G, Busonero F, Maschio A, Cusano R, Cuccuru G, Deidda F, Poddie F, Farina G, Dei M, Virdis F, Olla S, Satta Ma, Pani M, Delitala A, Cocco E, Frau J, Coghe G, Lorefice L, Fenu G, Ferrigno P, Ban M, Barizzone N, Leone M, Guerini Fr, Piga M, Firinu D, Kockum I, Lima Bomfim I, Olsson T, Alfredsson L, Suarez A, Carreira Pe, CastilloPalma Mj, Marcus Jh, Congia M, Angius A, Melis M, Gonzalez A, Alarcón Riquelme Me, da Silva Bm, Marchini M, Danieli Mg, Del Giacco S, Mathieu A, Pani A, Montgomery Sb, Rosati G, Hillert J, Sawcer S, D'Alfonso S, Todd Ja, Novembre J, Abecasis Gr, Whalen Mb, Marrosu Mg, Meloni A, Sanna S, Gorospe M, Schlessinger D, Fiorillo E, Zoledziewska M, and Cucca F. 2017. Overexpression of the Cytokine BAFF and Autoimmunity Risk. The New England journal of medicine 376:1615-1626. DOI 10.1056/NEJMoa1610528

Ritchie Me, Phipson B, Wu D, Hu Y, Law Cw, Shi W, and Smyth Gk. 2015. limma powers differential expression 
365

366

367

368

369

370

371

372

373

374

375

376

377

378

379

380

381

382

383

384

385

386

387

388

389

390

391

392

393

394

analyses for RNA-sequencing and microarray studies. Nucleic Acids Research 43:e47. DOI 10.1093/nar/gkv007

Cairns P. 2010. Renal cell carcinoma. Cancer biomarkers : section A of Disease markers 9:461-473. DOI 10.3233/CBM-2011-0176

Shannon P, Markiel A, Ozier O, Baliga Ns, Wang Jt, Ramage D, Amin N, Schwikowski B, and Ideker T. 2003. Cytoscape: a software environment for integrated models of biomolecular interaction networks. Genome Research 13:2498-2504. DOI 10.1101/gr.1239303

Siegel Rl, Miller Kd, and Jemal A. 2017. Cancer Statistics, 2017. CA: A Cancer Journal for Clinicians 67:7-30. DOI $10.3322 /$ caac. 21387

Chevrier S, Levine Jh, Zanotelli Vrt, Silina K, Schulz D, Bacac M, Ries Ch, Ailles L, Jewett Mas, Moch H, van den Broek M, Beisel C, Stadler Mb, Gedye C, Reis B, Pe'er D, and Bodenmiller B. 2017. An Immune Atlas of Clear Cell Renal Cell Carcinoma. Cell 169:736-749.e718. DOI 10.1016/j.cell.2017.04.016

Yang S, Liu T, Nan H, Wang Y, Chen H, Zhang X, Zhang Y, Shen B, Qian P, Xu S, Sui J, and Liang G. 2019. Comprehensive analysis of prognostic immune-related genes in the tumor microenvironment of cutaneous melanoma. Journal of Cellular Physiology. DOI 10.1002/jcp.29018

Aravind Subramanian, Pablo Tamayo, Vamsi K. Mootha, Sayan Mukherjee, Benjamin L. Ebert, Michael A. Gillette, Amanda Paulovich, Scott L. Pomeroy, Todd R. Golub, Eric S. Lander, and Jill P. Mesirov. 2005. Gene set enrichment analysis: A knowledge-based approach for interpreting genome-wide expression profiles. Proceedings of the National Academy of Sciences 102:15545-15550. DOI 10.1073/pnas.0506580102

Li T, Fan J, Wang B, Traugh N, Chen Q, Liu Js, Li B, and Liu Xs. 2017. TIMER: A Web Server for Comprehensive Analysis of Tumor-Infiltrating Immune Cells. Cancer Research 77:e108-e110. DOI 10.1158/00085472.can-17-0307

Capitanio U, and Montorsi F. 2016. Renal cancer. Lancet (London, England) 387:894-906. DOI 10.1016/S01406736(15)00046-X

Cremasco V, Woodruff Mc, Onder L, Cupovic J, Nieves-Bonilla Jm, Schildberg Fa, Chang J, Cremasco F, Harvey $\mathrm{Cj}$, Wucherpfennig K, Ludewig B, Carroll Mc, and Turley Sj. 2014. B cell homeostasis and follicle confines are governed by fibroblastic reticular cells. Nature Immunology 15:973-981. DOI 10.1038/ni.2965

Pelekanou V, Notas G, Theodoropoulou K, Kampa M, Takos D, Alexaki Vi, Radojicic J, Sofras F, Tsapis A, Stathopoulos En, and Castanas E. 2011. Detection of the TNFSF members BAFF, APRIL, TWEAK and their receptors in normal kidney and renal cell carcinomas. Analytical cellular pathology (Amsterdam) 

Medicine. DOI 12(10):12057-12067.

399 


\section{Figure 1}

\section{Work flow used in this analysis $\square$}

\section{The flow chart reflects the research idea of this study.}

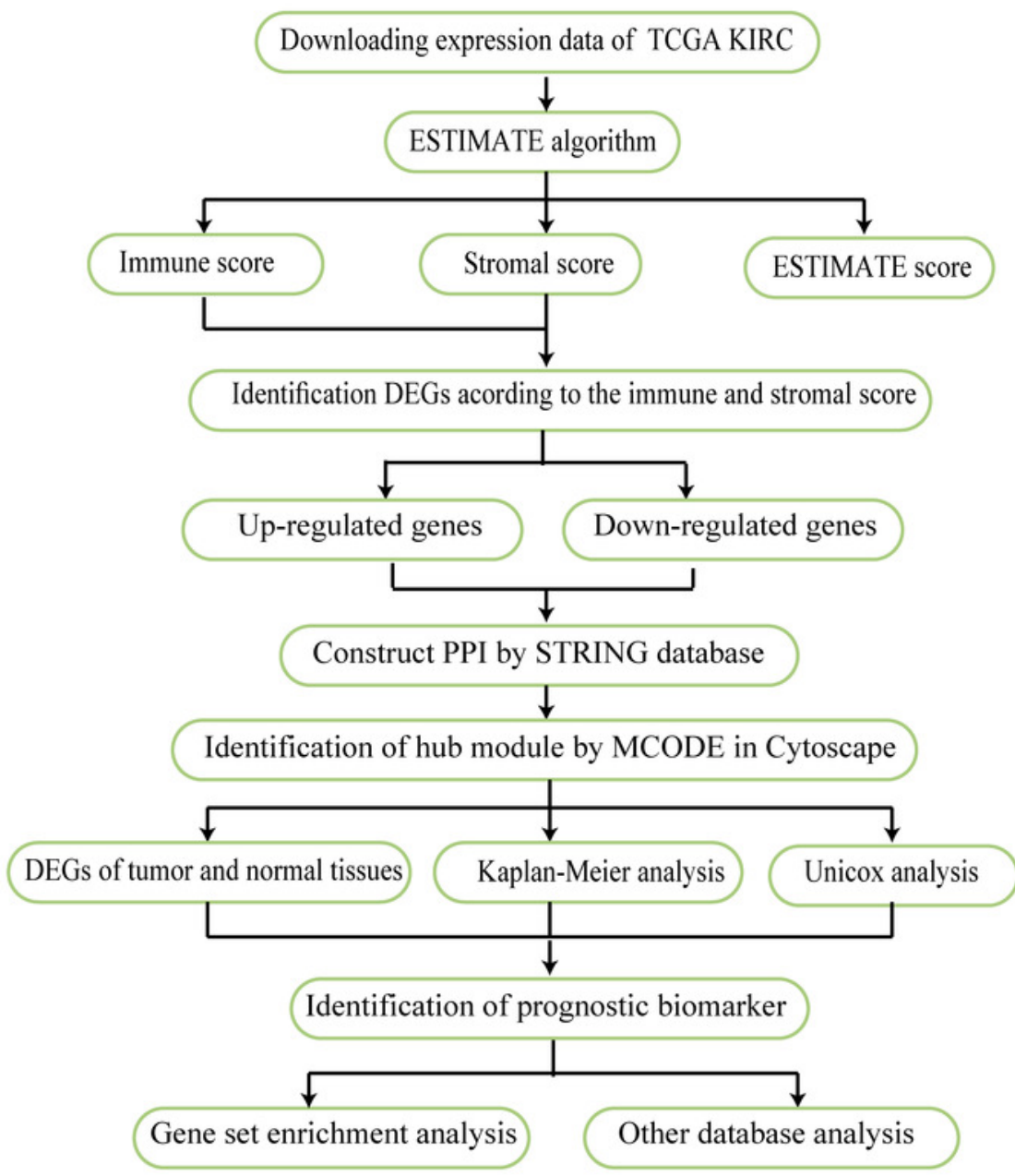


Figure 2

The scores obtained from the ESTIMATE algorithm were related to the KIRC overall survival rate and stage.

(A) Kaplan-Meier analysis of immune score. A value of $p<0.05$ means it is statistically significant. Cl: confidence interval (B) Stromal score. (C) ESTIMATE score. (D) Box diagram of immune score in different stages. Kruskal-Wallis test was used for comparison among groups. A value of $p<0.05$ indicates that it is statistically significant. (E) Stromal score. (F) ESTIMATE score.

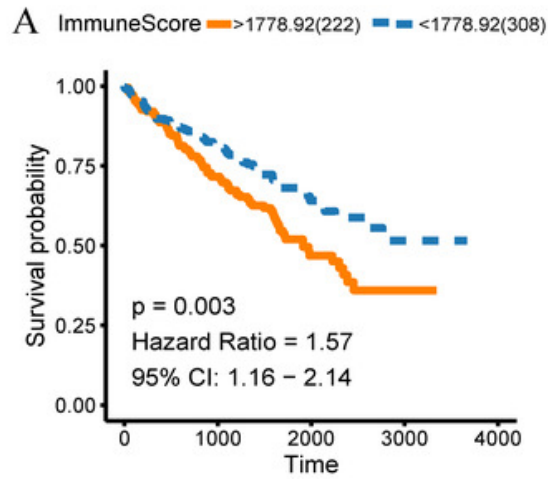

$\mathrm{D}$

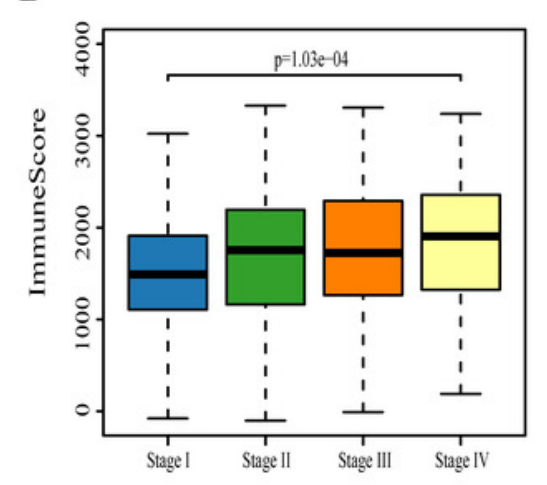

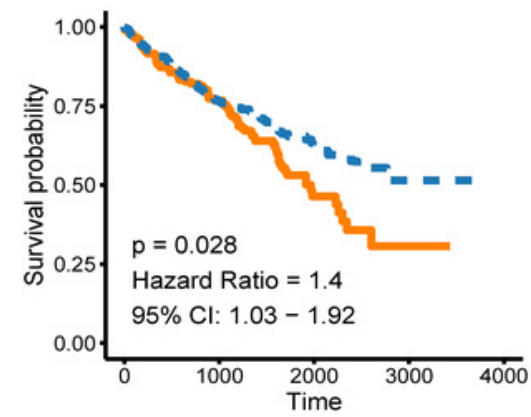

$\mathrm{E}$

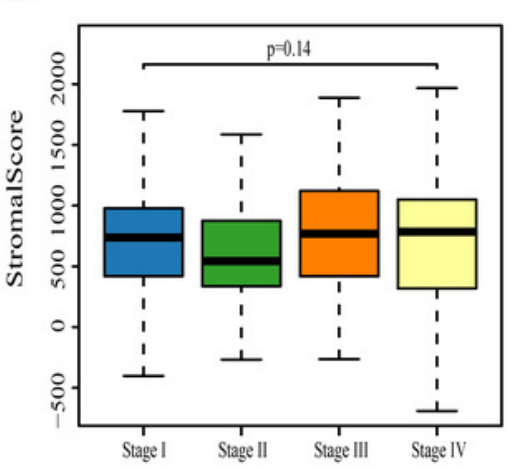

C ESTIMATEScore $=>2785.69(179)==<2785.69(351)$

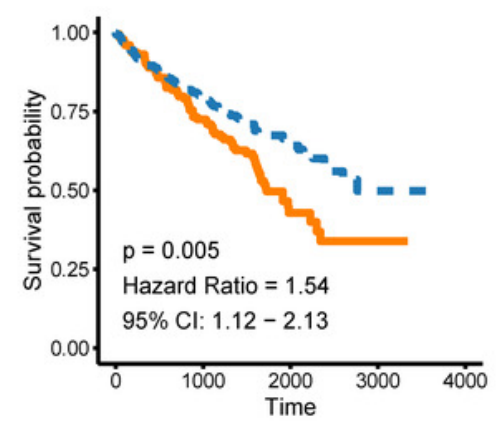

$\mathrm{F}$

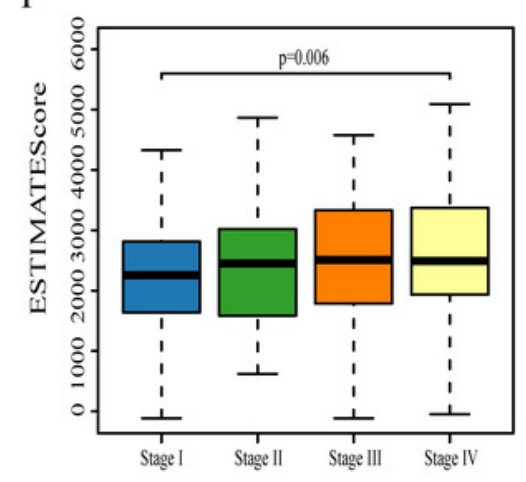




\section{Figure 3}

Comparison of expression profiles for genes with low or high immune and stromal scores.

(A) The heatmap of DEGs between high and low immune scores. (B)The heatmap of DEGs between high and low stromal scores. (C) Venn diagram shows the number of up-regulated DEGs. (D) Venn diagram shows the number of down-regulated DEGs. (E) A bubble chart shows the top 20 enriched GO terms. The GO analysis had an FDR of less than 0. 05. (F) The KEGG enrichment analysis exhibited an FDR of the six pathways that were less than 0.05 . 
A

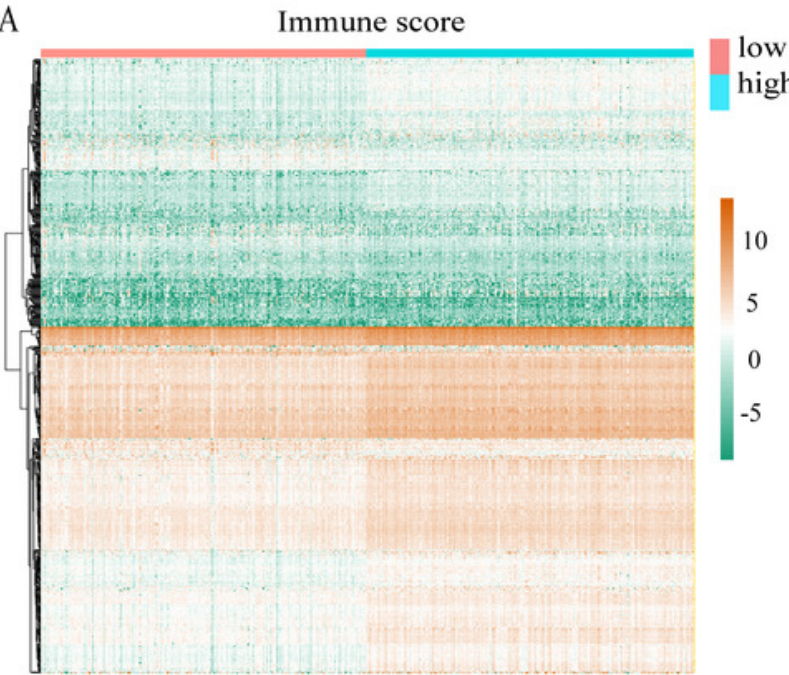

C
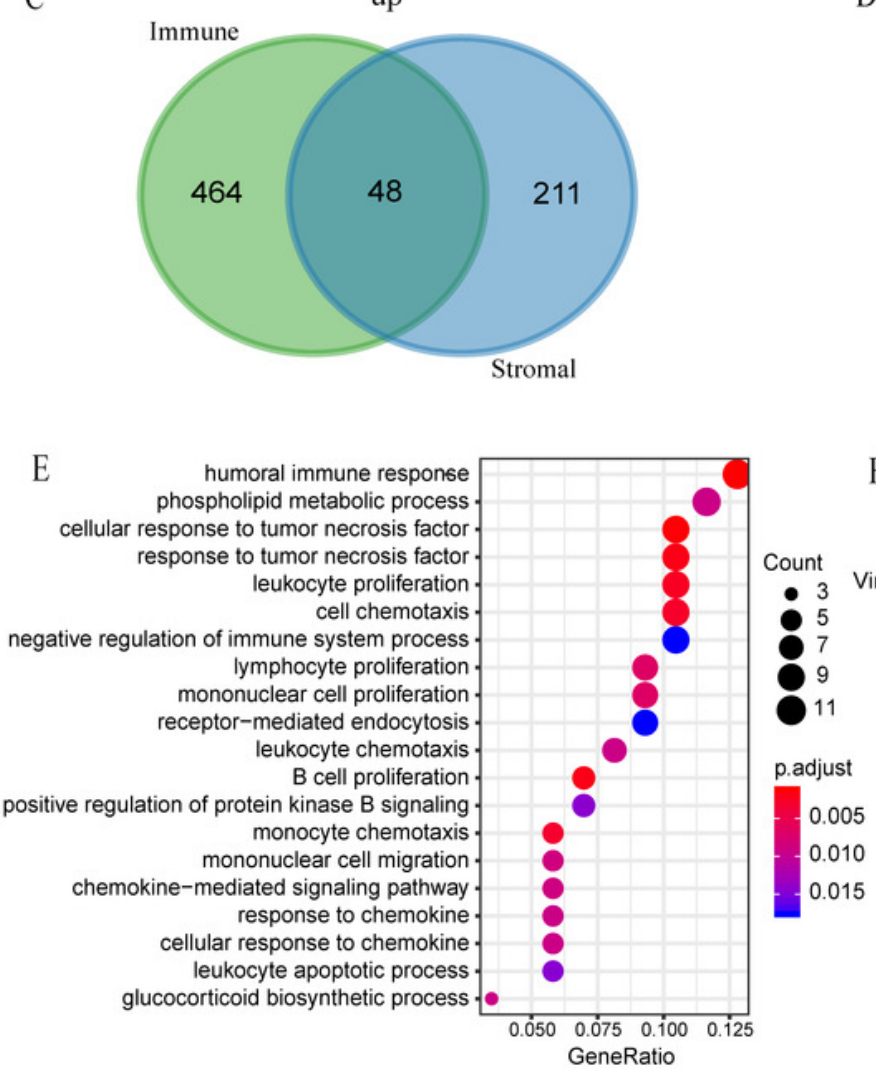

B

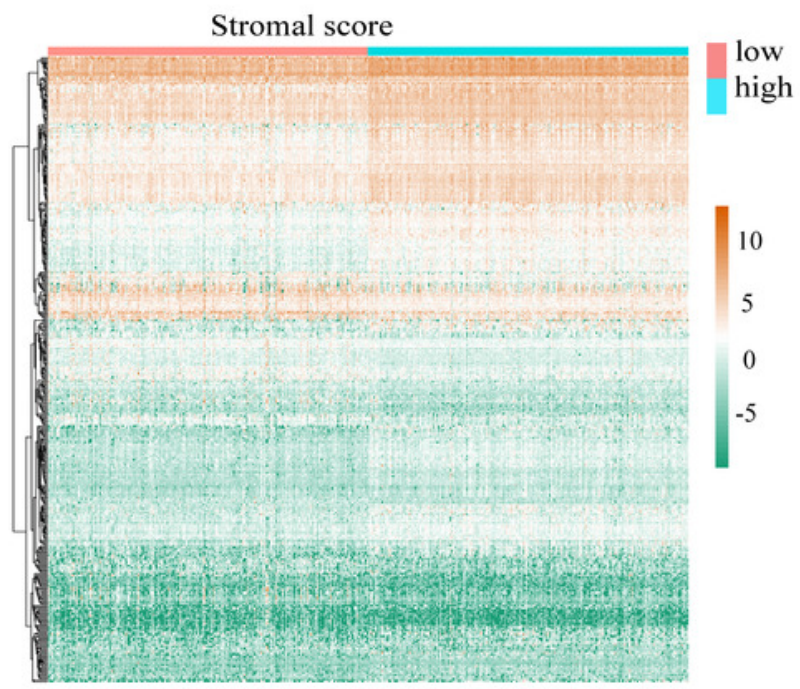

D

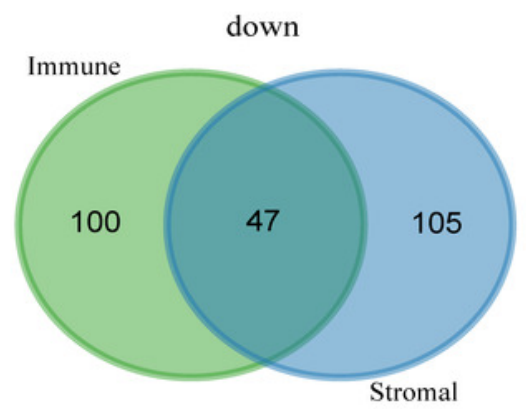

F

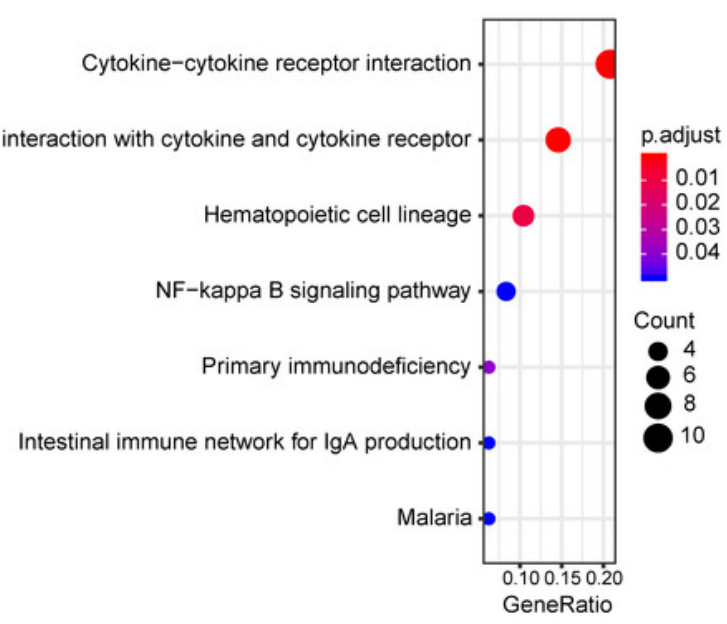


Figure 4

Construction of PPI network and identification of clusters.

(A) The PPI network. (B) Cluster 1. (C) Cluster 2. (D) Cluster 3. (E) Cluster 4.

A

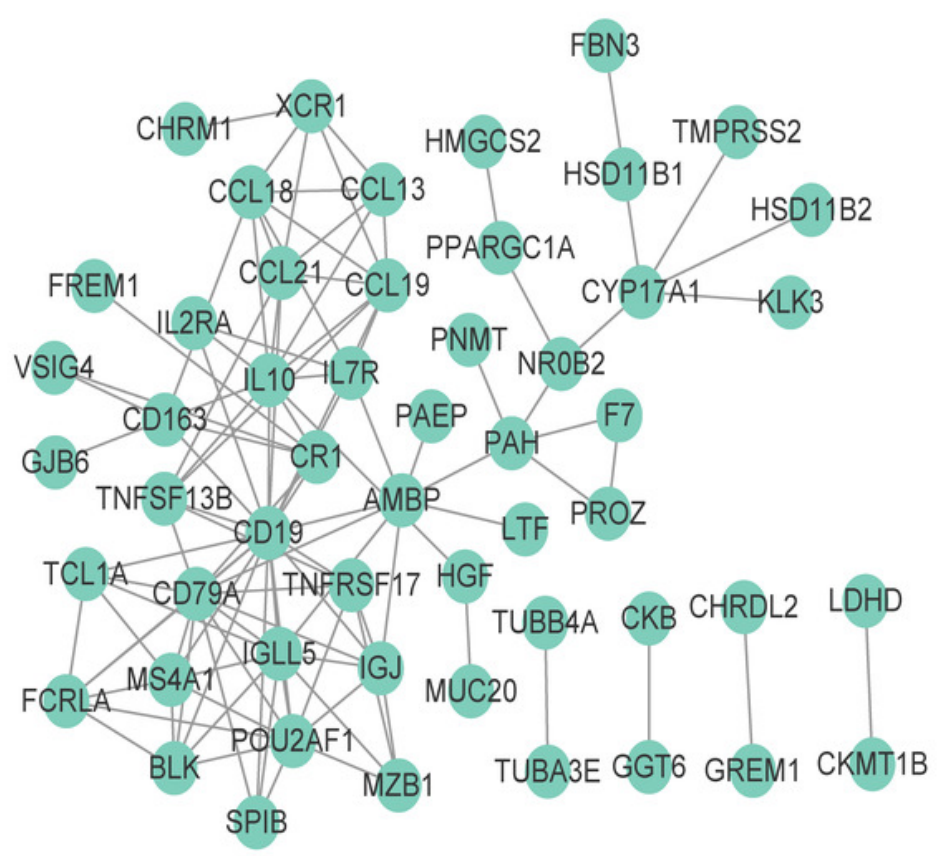

B

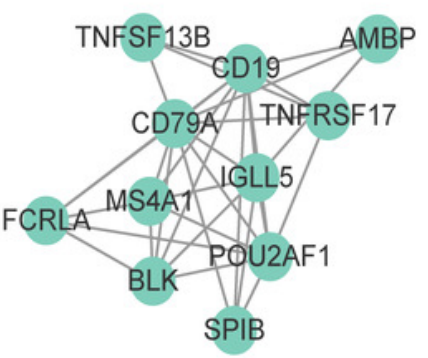

C

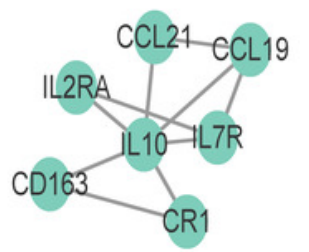

D

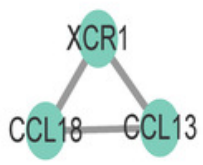

E

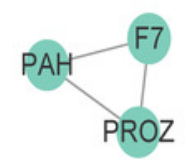




\section{Figure 5}

Venn analysis of results from three analyses.

Blue circles indicate differentially expressed genes of the tumor and normal tissue $(|\log F C>1|, P<0.05)$. Red circles indicate genes with significant results from univariate analysis $(p<0.05)$. Green circles mean genes with significant results from Kaplan-Meier analysis $(p<0.05)$.

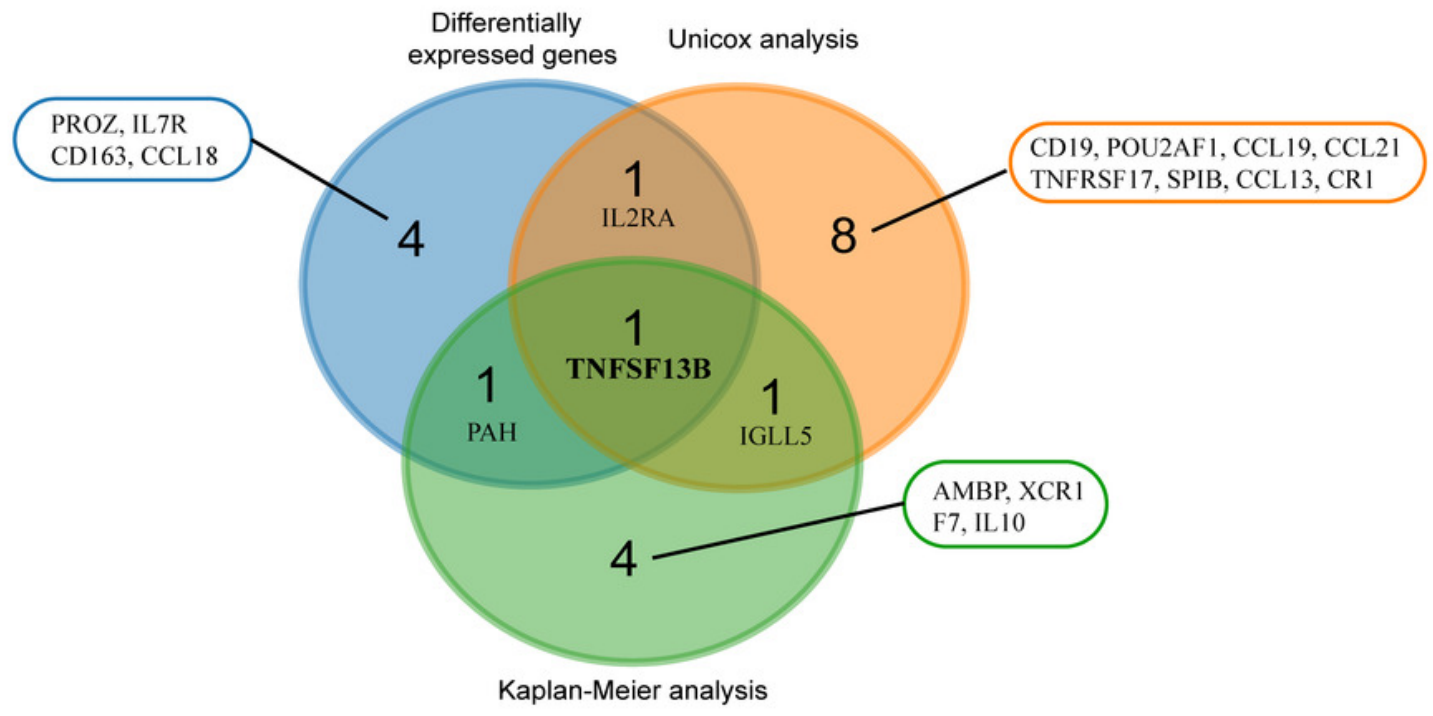




\section{Figure 6}

Analysis of the relationship between TNFS13B and clinical factors.

(A) Scatter plot of TNFS13B expression in tumor and normal tissues. Wilcoxon test was used

to compare the two groups. (B) Kaplan-Meier analysis of TNFS13B. (C) Forest plot of univariate COX regression analysis for clinical factors. (D) Forest plot of multivariate COX regression for clinical factors. (E-D) Box diagram of TNFSF13B expression under different grades and stages. Kruskal-Wallis test was used for comparison among groups. A value of $p<0.05$ indicates that it is statistically significant. 
A

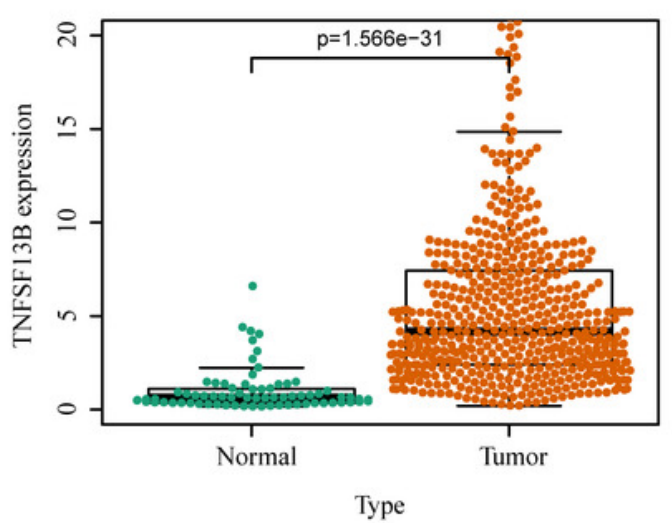

C

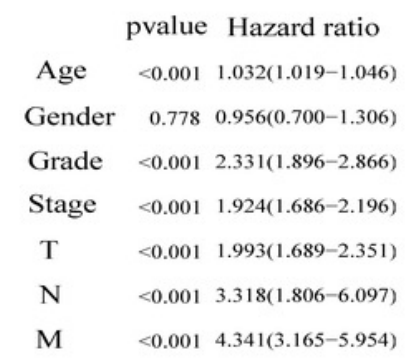

TNFSF13B $<0.001 \quad 1.049(1.024-1.074)$

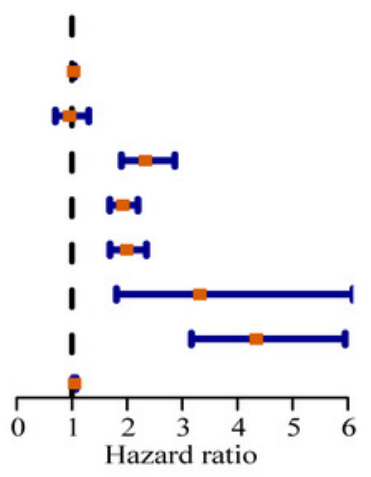

E

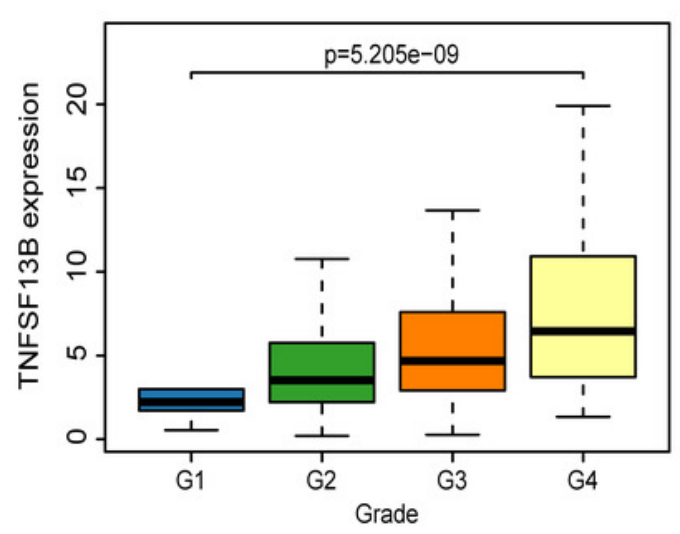

B

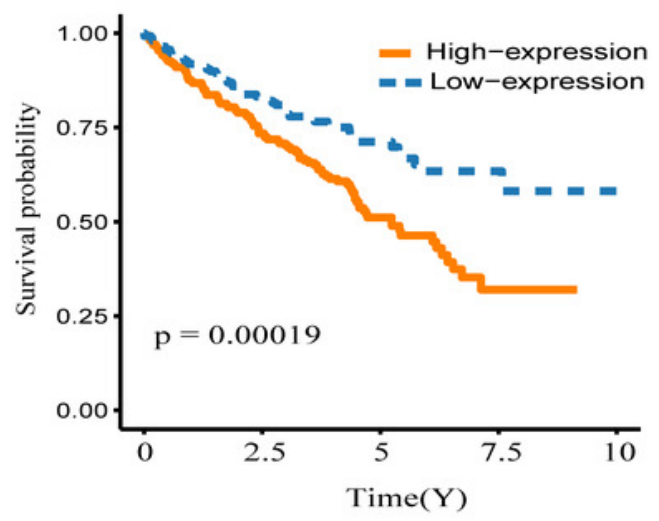

D

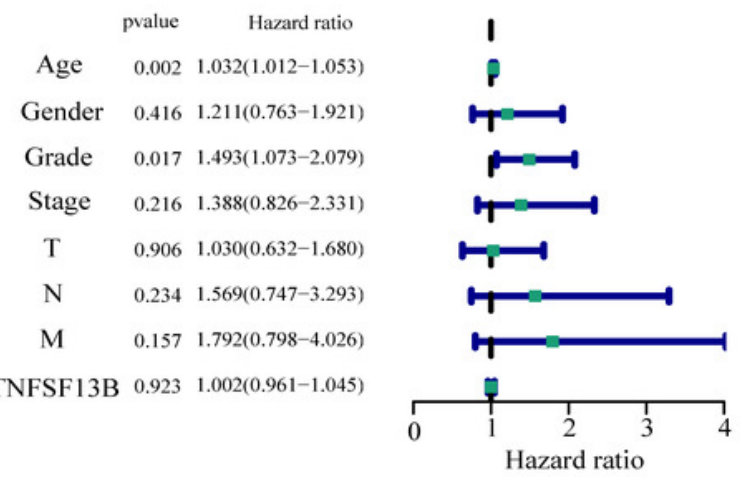

F

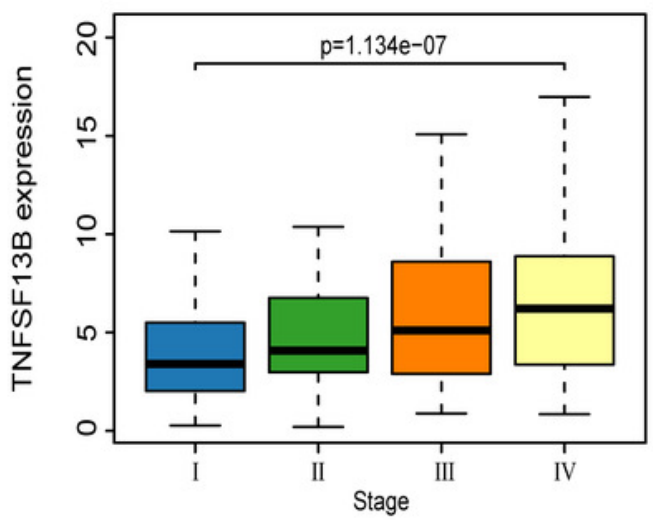


Figure 7

Tumor microenvironment exploration of TNFSF13B.

(A) Correlation scatters diagram of the immune score and TNFSF13B expression. (B) Stromal score. (C) ESTIMATE score. (D) Correlation scatters Diagram of Tumor purity and TNFSF13B expression. (E) B cell. (F) CD8+ T cell. (G) CD4+ T cell. (H) Macrophage. (I) Neutrophil. (J) Dendritic cell. A value of $p<0.05$ indicates that it is statistically.
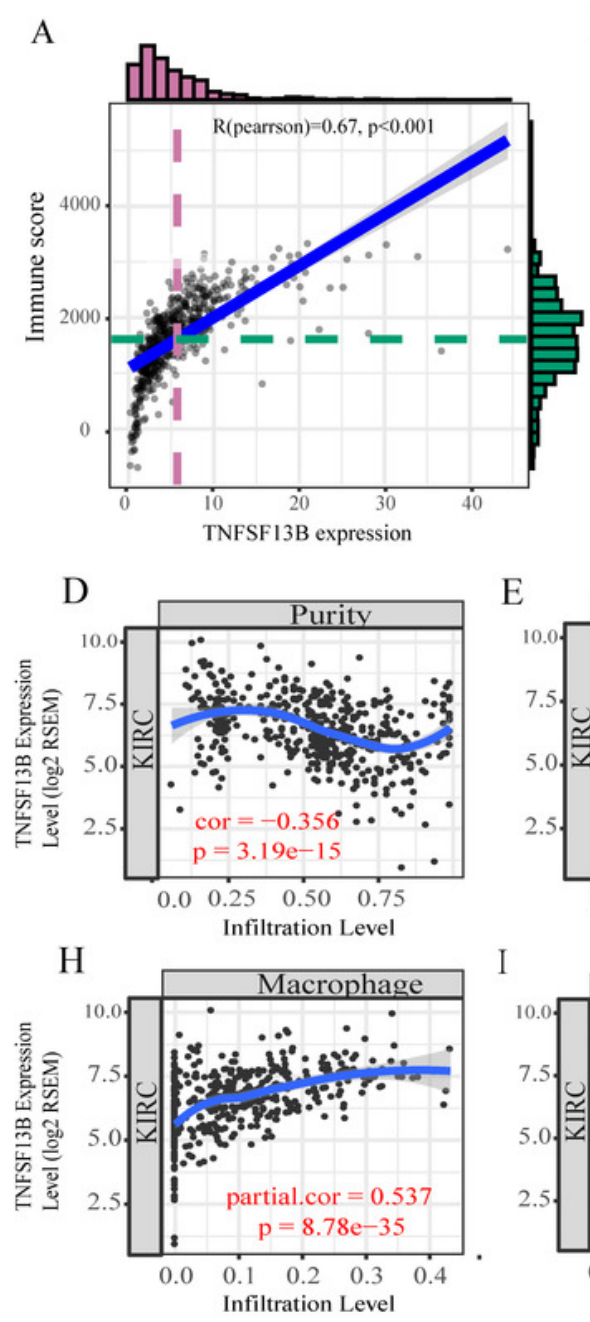

B
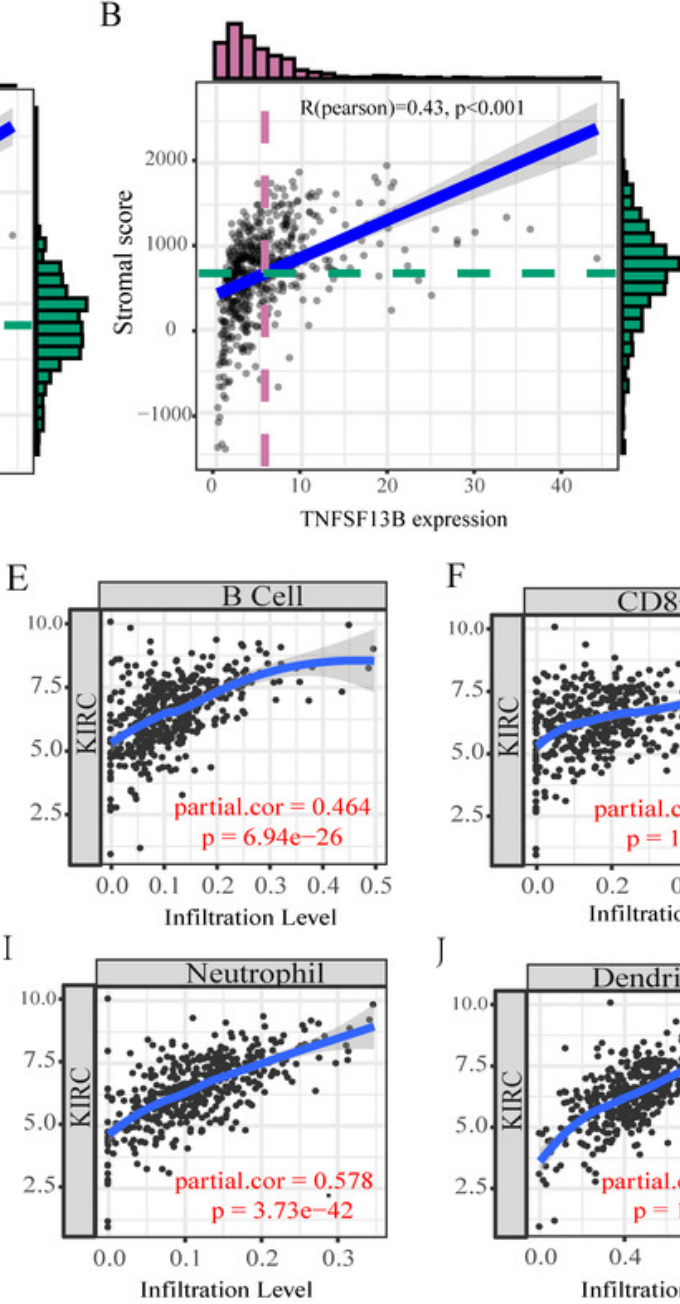

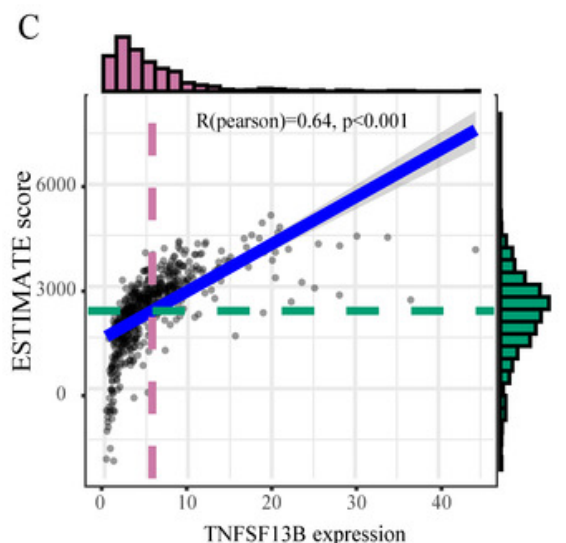

TNFSF13B expression

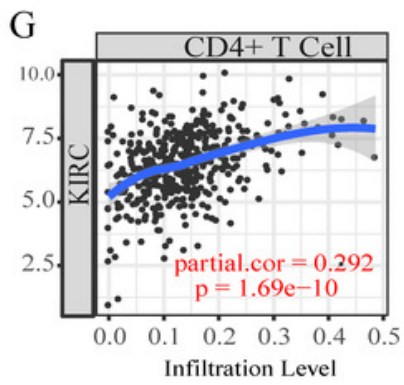

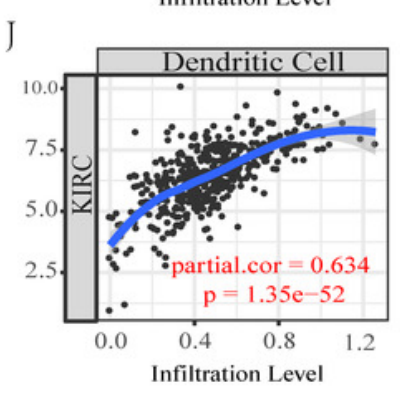

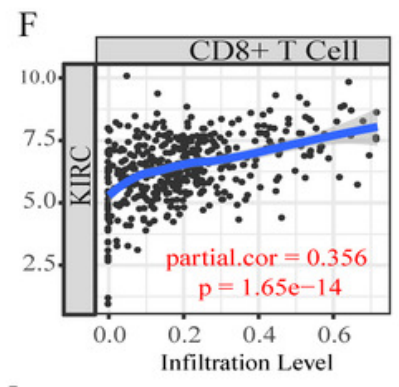


Figure 8

Gene set enrichment analysis and drug target network.

(A) A broken line above represents an enrichment pathway, and the straight line below

represents every gene in the pathway. (B) Network diagram of drugs and acting genes.
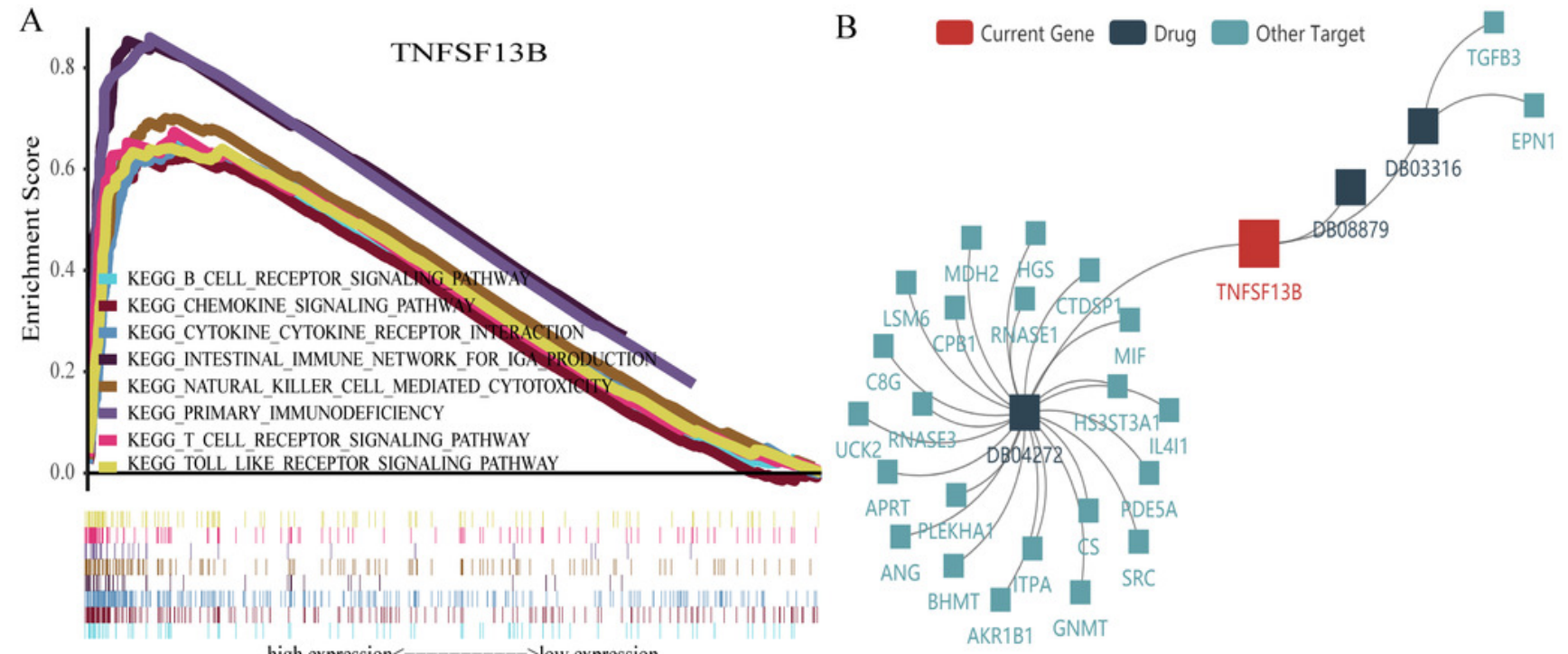